\title{
PROCESSES AND SYSTEMS Clinician-directed improvement in the accuracy of hospital clinical coding
}

\author{
Authors: Krsna Mahbubani, ${ }^{\mathrm{A}}$ Fanourios Georgiades, ${ }^{\mathrm{B}}$ En Lin Goh, ${ }^{\mathrm{C}}$ Swathikan Chidambaram, ${ }^{\mathrm{D}}$ \\ Prasanthi Sivakumaran, ${ }^{\mathrm{E}}$ Timothy Rawson, ${ }^{\mathrm{F}}$ Sucharita Ray, ${ }^{\mathrm{G}}$ Anita Hudovsky ${ }^{\mathrm{H}}$ and Dipender Gill ${ }^{\mathrm{I}}$
}

'Payment by results' (PbR) remuneration for healthcare services relies on the accurate conversion of diagnoses into Healthcare Resource Group (HRG) codes that are then reimbursed. Inconsistencies in documentation can result in inaccuracies in this process, with consequent implications for measuring activity, disease incidence and organisational performance. The aim of this study was to determine if clinician involvement increases accuracy in the coding of medical cases. Selected records of medical patients admitted to a London NHS trust between November and December 2016 were reviewed by a coding auditor and a clinician. Any changes to the codes and HRG tariff were noted. In total, 123 cases were considered. Changes in code were made on 68 instances, resulting in an overall increase in remuneration of $£ 39,215$; an average of $€ 318$ per patient. The primary HRG code was changed in 31 cases which accounted for $£ 28,040$ of the increase in tariff. In conclusion, clinician involvement can help with documentation ambiguities, thus improving the accuracy of the coding process in a medical setting. Although such collaborative working offers advantages for both the clinician and the coding team, further work is required to investigate the feasibility of this recommendation on a larger scale.

Keywords: Payment by results, clinical coding

\section{Introduction}

The financing of the NHS has undergone numerous reforms. In 2004, the Department of Health introduced a tariff-based system

Authors: ${ }^{\text {A }}$ linical coding fellow, St Mary's Hospital, London, UK; ${ }^{B}$ foundation doctor, Imperial College Healthcare NHS Trust, London, UK; ' medical student, Imperial College London, London, UK; ${ }^{D}$ medical student, Imperial College London, London, UK; ${ }^{E}$ medical student, Imperial College London, London, UK; ${ }^{{ }^{F}}$ clinical research training fellow, Imperial College London, London, UK; ${ }^{G}$ clinical coding quality performance manager, Imperial College Healthcare NHS Trust, London, UK; ${ }^{H}$ head of clinical coding services, Imperial College Healthcare NHS Trust, London, UK; ${ }^{\mathrm{I}}$ clinical research training fellow, Imperial College Healthcare NHS Trust, London, UK termed 'Payment by results' (PbR), which reimburses hospitals for services provided. ${ }^{1}$ The clinical information is translated into codes based on the 10th International Statistical Classification of Diseases and Related Health Problems (ICD-10) and the Office of Population Censuses and Surveys Classification of Interventions and Procedures version four (OPCS-4). These codes are subsequently converted into specific Healthcare Resource Groups (HRG). ${ }^{2}$ The HRG codes represent a group of diagnoses and interventions that use a similar amount of NHS resources, and determine the final tariff received by the trust (Fig 1). PbR aims to correct ineffective payment structures and create a more costefficient organisation, potentially leading to a reduction in waiting times, increased productivity and better use of capacity. ${ }^{3}$ However, this is limited by many factors, particularly the inconsistency in the coding process. ${ }^{4-6}$

Following discharge of a patient from hospital, patient reports are submitted for coding. ${ }^{4,5}$ While all inpatient spells are consequently coded, a fraction are also audited to optimise precision. ${ }^{7}$ Accurate clinical coding is important for several reasons. Firstly, it ensures that the range of clinical activity being undertaken is reliably captured to further guide resource allocation in proportion to demand. Secondly, it also has academic implications towards understanding disease incidence and prevalence, along with geographical differences and temporal trends in health behaviour at local, national and international levels. Finally, clinical coding also allows for a measure of performance, as comparisons can be made in terms of clinical and financial outcomes resulting from the management of the same medical conditions.

There are several key factors that determine the accuracy of clinical coding. These include personnel used for the coding process, ${ }^{5-7}$ ambiguity in documentation, ${ }^{6-8}$ clinical knowledge to distinguish between codes and clinical experience. ${ }^{8-13}$ All clinical coders receive rigorous training and are required to pass a national accreditation exam. However, the rules and standards of the classifications mean that coders cannot infer or assume clinical meaning and must strictly interpret the information as per their training. This can lead to a clinical condition not being recorded. To this end, coding performance may be improved with clinician involvement. ${ }^{7,8,11}$ This study aimed to determine the impact of clinician input on the accuracy of the coding process in an acute medical setting. We were interested in assessing how the involvement of a clinician could help address any documentation ambiguities faced by the coding team and how this impacted the 
Fig 1. A schematic representation of the coding process. HRG $=$ Healthcare Resource Group

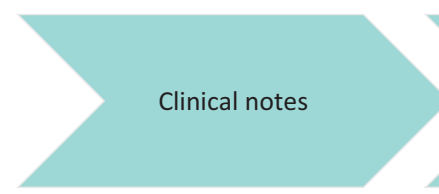

Eg ward round entries, discharge summary, investigation reports

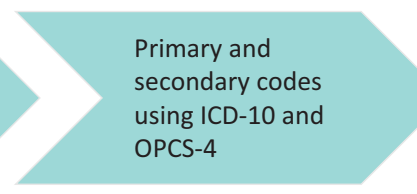

Eg ICD-10 I21.9 for acute myocardial infarction (unspecified) and OPCS-4 X501 for direct current cardioversion

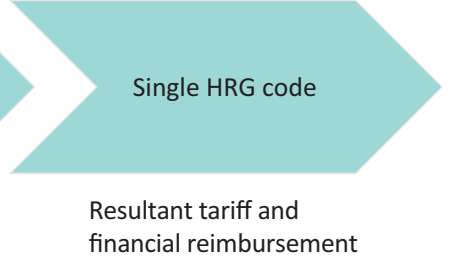

financial reimbursement final HRG tariff. The a priori hypothesis was that clinician input would result in more equitable funding for the services provided.

\section{Methods}

For this pilot study, the clinical coding team identified a sample of medical patients that were discharged between November and December 2016 from Hammersmith Hospital, Charing Cross Hospital or St Mary's Hospital, all of which are part of the Imperial College Healthcare NHS Trust. The sample of patients was selected using the 'Liveaudit' software (Woodward Associates (UK) Ltd). This program identifies patients where audit of clinical coding is likely to further enrich data for greater depth and clinical accuracy, alongside correct income reimbursement. The software trawls cases to identify a sample where, for example:

> HRG analysis has previously identified coding improvements to be more likely

$>$ the length of stay exceeds that expected for the given diagnosis

$>$ there are inconsistencies in comorbidity recording

$>$ the most complex diagnoses are being observed.

The data from the coding team for each patient admission were initially received and retrospectively analysed by a fully registered doctor with the General Medical Council (GMC) of the UK who has completed 2 years of basic training. Prior to commencing this project, the doctor received a basic induction lasting 2 hours on the clinical doing process, while observing examples of this process being undertaken. Reviews were performed within 4 weeks of the patient discharge date, in keeping with the timeline for submission of results for financial reimbursement. All notes were reviewed jointly by the coding auditors and clinician, based on the ICD-10 and OPCS- 4 systems, using a combination of clinical notes, discharge summaries and investigation results. The primary and secondary codes were compared against the original codes to detect any errors.

All data, including the patient demographics, ICD-10 codes for primary and secondary diagnoses, OPCS- 4 codes for primary and secondary procedures, and HRG codes were extracted. The payment received after the changes were made was subsequently calculated. This was directly compared to the initial tariff allocated. All data were entered into Microsoft Excel (Microsoft, Washington, USA) for statistical analysis. Parametric data are summarised by means and standard deviations.

This work was undertaken as a part of a locally approved quality improvement project and further ethical approval was not required.

\section{Results}

\section{Patient demographics}

On review of patient notes, a total of 123 cases were identified in the initial audit from the three hospitals. The data included 61 males $(49.6 \%)$ and 62 females $(50.4 \%)$, with the mean age of the participants 65.9 years (SD 7.8 years).

\section{Tariff changes and coding interpretation}

Of the 123 cases reviewed, changes were made in 68 instances $(55.3 \%)$. The overall increase in remuneration for the trust was $£ 39,215$. This equates to an increase of $£ 318$ per patient. The initial primary code was changed in 31 cases $(25.2 \%)$, resulting in an increase in tariff of $£ 28,040$. In 11 of the 31 cases, there were additional changes made to the secondary codes and procedures. There were only 11 instances when a change in the secondary diagnosis alone changed the tariff, and the total increase in tariff from these cases was $£ 11,175$. A change to the procedure alone generated no income change.

Out of the 123 cases reviewed by the coding team a symptom was put as the primary diagnosis in 21 cases (17\%). 'Dyspnoea' (five instances), 'confusion' (two instances) and 'chest pain' (two instances) were identified as the top terms used. Of these, the original code was altered by the clinician and coding auditors in 14 cases resulting in an increase in the tariff of $£ 15,683$. Of the 10 changes that resulted in the maximum increase in tariff, five were due to a change from a symptom to diagnosis (Table 1).

In 44 of the 123 records ( $35.8 \%$ ), a secondary diagnosis code was present and subsequently changed. In only four of these cases, the secondary diagnoses were purely related to past medical history. Notably, most secondary diagnoses were conditions that patients experienced during their stay in the hospital, but were not present on admission. Abnormal blood results such as 'hyponatraemia', 'acute kidney injury' (AKI) and 'type 1 respiratory failure' (T1RF) were most commonly missed (Figure 2). In three out of the five cases where AKI had been missed, there was no mention of this in the discharge summary. The addition of these secondary complications also resulted in a significant increase in the tariff. For example, the addition of T1RF in one case resulted in an additional $£ 1,958$ remunerated to the trust (Table 1).

\section{Discussion}

The PbR scheme aims to ensure that healthcare resources are utilised in the most fair and equitable manner by using 
Table 1. The 10 coding improvements that most

increased tariff

\begin{tabular}{|c|c|c|}
\hline $\begin{array}{l}\text { Change to primary } \\
\text { diagnosis }\end{array}$ & $\begin{array}{l}\text { Addition of secondary } \\
\text { diagnoses }\end{array}$ & $\begin{array}{l}\text { Increase in } \\
\text { tariff }(€)\end{array}$ \\
\hline Delirium $\rightarrow$ UTI & Constipation & 4,475 \\
\hline Dementia $\rightarrow$ UTI & Constipation, AKI & 4,475 \\
\hline Hallucinations $\rightarrow$ UTI & None added & 4,475 \\
\hline Fall $\rightarrow$ UTI & None added & 2,992 \\
\hline $\begin{array}{l}\text { Liver failure } \rightarrow \text { liver } \\
\text { cirrhosis }\end{array}$ & $\begin{array}{l}\text { T1RF, orbital floor } \\
\text { fracture, candida }\end{array}$ & 1,822 \\
\hline Not changed & Hypocalcaemia, T1RF & 1,958 \\
\hline Not changed & AKI, obesity & 1,958 \\
\hline Seizure $\rightarrow$ peritonitis & None added & 1,629 \\
\hline Pneumonia $\rightarrow$ CCF & None added & 1,390 \\
\hline Pain $\rightarrow$ gout & Pan-systolic murmur & 1,339 \\
\hline
\end{tabular}

AKI = acute kidney injury; $C C F$ = congestive cardiac failure; UTI = urinary tract infection; T1RF = type 1 respiratory failure

the outcomes of the care provided to guide monetary reimbursement. ${ }^{14}$ Achieving this requires an accurate clinical coding process that translates the diagnoses and procedures into HRG codes. Previous studies have reported a wide range of accuracy rates, ${ }^{15}$ ranging from just $52.2 \%{ }^{13}$ to $98.0 \%{ }^{16}$ This was dependent on hospital and specialty. ${ }^{17}$ Furthermore, these studies have predominantly involved senior clinicians typically at consultant grade; yet it is more junior doctors who are primarily involved in the clinical documentation and interaction with patients. $^{18}$

It is evident that good documentation of patient information by clinicians will minimise coding errors and optimise reimbursement.
Shaihid and Tindal reported that mistakes in the documentation of orthopaedic procedures caused an annual average loss of $£ 118,056$. $^{6}$ This was corroborated by Nouraei et al in their analysis of 8,888 discharge summaries of emergency medical admissions, which identified at least one error in $55 \%$ of cases, equating to a loss of $£ 816,977$ over 3 years. ${ }^{7}$ From these findings, the authors concluded that information transferred between clinicians to coders is prone to subjectivity and error. While this reduces the accuracy of coding, clinician involvement can go some way in overcoming this problem.

From our results, the involvement of a fully registered doctor with the GMC of the UK who has completed 2 years of basic clinical training improved the accuracy of the coding process. The clinician helped the coders with the auditing workload and could provide help regarding medical issues and poorly documented diagnoses, while the auditors could teach the clinician about the coding process and inform them of the key problems faced. This resulted in a higher remuneration equating to an increase of $€ 318$ per patient. This supports previous work that has shown clinician input to have similar beneficial effects. ${ }^{19,20}$ However, such benefits are not only limited to financial rewards. ${ }^{11,15,19,20}$ Accurate coding also offers benefit towards hospital governance, in terms of understanding the underlying activity so that resources may be better allocated and organised. ${ }^{11,19,20}$ Similarly, there are wider implications, at national and international level, ${ }^{15}$ as such codes are also used to estimate disease incidence and prevalence along with temporal and geographical variation. Indeed, such benefits afforded by precise coding would survive any change in hospital financing away from a PbR system.

Our study has highlighted the value of clinician input in several ways. For example, one of largest increases in tariff $(£ 4,475)$ was due to a change from a diagnosis of 'hallucinations' to 'urinary tract infection'. The discharge summary had stated that the diagnosis was delirium. Although this is clinically correct, accurate coding requires the cause of the delirium to be identified where

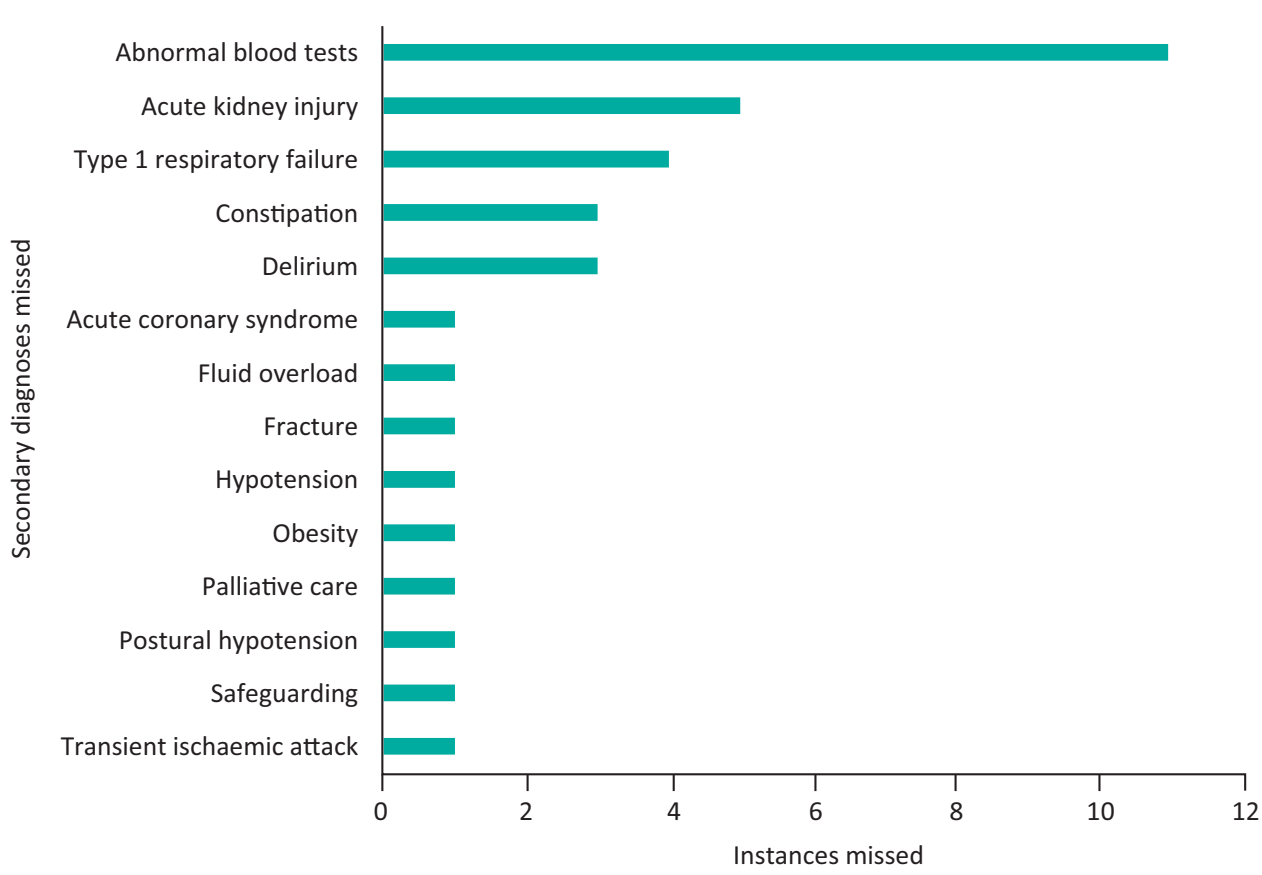

Fig 2. Bar chart depicting common secondary diagnoses missed from the original coding. 
possible. Some modifications were less easily identifiable from the patients' notes. In one case, a change in primary diagnosis from 'dyspnoea' to 'aortic stenosis' was made. The discharge summary simply stated 'shortness of breath' and never specified a cause for this. Nevertheless, an echocardiogram had demonstrated aortic stenosis and cardiology follow-up had been arranged for this. It became apparent that the symptoms were related to the patient's aortic stenosis and appropriate acknowledgement of this generated a significant increase in the tariff. This would not have been possible without a clinician's input.

Following from this, clinician input was also essential to interpret investigation results that had not been explicitly stated. The most important complications of inpatient stay from a coding perspective include AKI, T1RF and delirium. Addition of these diagnoses can have a significant impact on the tariff; however, coders are unable to interpret investigation results unless they have been documented by a clinician. For example, in one case the doctors documented a partial pressure of oxygen of $6.6 \mathrm{kPa}$ when breathing room air, but the term T1RF was not used in any of the notes or the discharge summary. When this diagnosis was eventually accounted for in the coding, the tariff increased by $£ 1,958$. These data highlight both poor documentation practice as well as the resulting 'coder constraint', whereby coders cannot attribute codes to a case due to ambiguous and non-specific information. ${ }^{9}$

Documentation and coding accuracy are closely linked ${ }^{7}$ and this study has reinforced that poor clinical documentation is a major source of coding inaccuracy. For example, this is not only highlighted by the poor documentation of complications such as AKI and T1RF, but also by the finding that modification of the primary diagnosis from a symptom to a diagnosis can have a significant impact on the tariff. Of note, in eight out of the 14 cases in which the symptom coded as the primary diagnosis was changed, the original symptom correlated with the main diagnosis documented in the discharge summary. Interestingly, Nouraei et al noted a 'diagnostic hesitancy' among more junior doctors whereby they often treat patients based on investigation examples rather than committing to a diagnosis. ${ }^{7}$ Indeed, it is noteworthy that more than one in six primary codes represents a symptom rather than diagnosis. A prime example of this is the use of 'troponin positive event' as opposed to 'myocardial infarction'. This makes it difficult for coders to assign a code for that condition. Therefore, having a clinician on the coding team can help overcome such problems. Where this is not always possible, a clear, concise and well-formulated discharge summary would also go a long way towards accurate coding. Junior clinicians can be trained in this with assistance from the coding department. ${ }^{11,18}$

In addition to documentation, the effectiveness of $\mathrm{PbR}$ relies on constantly updating tariffs to closely reflect the cost of the patient admission. In April 2017, a new HRG tariff system, 'HRG4+', was introduced with the aim of improving the accuracy of the coding process. ${ }^{21}$ One of the key changes in HRG4+ compared to its predecessor is the introduction of an interactive model of complexity and comorbidity splits, which make consideration of the cumulative comorbidities. ${ }^{21}$ It is also important to consider the system for funding the NHS in relation to reimbursement for clinical activity. Although the switch to the PbR system was shown to improve efficiency in the provision of healthcare, it essentially rewards an increased volume of activity. This payment system has limitations with regards to the rising population. For example, introduction of the national $30 \%$ marginal rate tariff in 2010 meant that hospitals were given less money for emergency admissions. ${ }^{22}$ In addition, some commissioners even introduced the idea of 'cap and collar' arrangements whereby they would not pay the trusts more than a finite amount despite higher levels of activity. ${ }^{22}$ The alternative to a volume-based payment system is one based on outcomes. This has been introduced in primary care with the Quality and Outcomes Framework (QoF). ${ }^{23}$ There have also been some payment by performance schemes introduced in secondary care, with variable outcomes. ${ }^{23}$ Examples include the best practice tariffs (BPT) introduced in 2010 to try and reduce the variation in clinical practice. For example, the best practice guidelines for stroke advise same day brain imaging and admission to a specialist stroke unit, with an added financial incentive for adhering to these criteria. Although there is scope for using such guidelines to improve quality and reduce the variation of care, the complexity of the reimbursement system means that adopting all of them may not provide a significant financial advantage for the hospitals. ${ }^{23}$ Ideally, there needs to be an effective combination of rewarding both quality and quantity, which is a work in progress. Finally, there may also be scope to utilise new technologies to optimise the clinical coding process, as demonstrated by Long et al in their development of a programme that extracts the diagnoses and procedures from the discharge summaries for coding. ${ }^{24}$

\section{Limitations and further work}

There is scope for further work in evaluating the benefits of utilising a clinician in the coding process. This pilot study was conducted over a short time frame of two winter months with a reasonably small sample size of cases. Furthermore, the patients were selected using software that predicted their audit would likely further improve coding accuracy, and it is therefore not clear whether these findings can be extrapolated more generally. Similarly, it remains to be clarified which grade of doctor is best suited to working with the coders to optimally improve accuracy. Although this study employed a fully registered doctor with the GMC of the UK who has completed two years of basic training, it may well be the case that there are greater gains to be made with utilisation of more senior clinicians, particular in view of the 'diagnostic hesitancy' observed among more junior doctors. In addition, it may be effective to focus on the specialties that require the most resources, where any restoration of correct remuneration will have the greatest impact. Further work on other interventions to improve the accuracy of the coding process is also required. These include education of clinicians about coding and the importance of clarity in medical records, implementation of a comorbidity checklist, review of diagnoses coded, and updating of the coding policy and procedure manual. As a measure of effect, a crossover study may be useful to investigate how the documentation practices of doctors change following education and training in clinical coding. From another perspective, there may also be gains to be made through greater clinical training of the coding team, so that they themselves also develop some of the expertise offered through clinician involvement.

\section{Conclusion}

Overall, the recommendation for inclusion of clinical staff involved in the patient journey as part of the coding process is a clear one, given the above results. Not only does the coding team 
benefit from the knowledge of the clinician, but the doctor also learns about the coding process, allowing them to improve their documentation appropriately in the future. However, further work is necessary to investigate the feasibility of this recommendation on a larger scale. In a healthcare system strained for resources, adding further responsibilities to clinicians will increase their workload, and may be counterproductive. It will be necessary to explore whether the efficiency savings offered by involving clinical staff in the coding process will outweigh the cost of their increased activity. Given the increasing strain on the health service with increasing demands, such quality improvement initiatives will be paramount towards ensuring sustainability.

\section{Conflict of interest}

All authors declare no conflict of interest.

\section{Author contributions}

$\mathrm{KM}, \mathrm{SR}, \mathrm{AH}$ and DG conceived the study objectives and design. KM, SR, AH collected data. KM, SR, AH and DG analysed the data. All authors interpreted the results. KM, FG, EG, SC, PS, TR, AH and DG drafted the manuscript. All authors revised the manuscript.

\section{References}

1 Department of Health. Reforming NHS financial flows: introducing payment by results. London: $\mathrm{DH} ; 2002$.

2 Busse R, Geissler A, Aaviksoo A et al. Diagnosis related groups in Europe: moving towards transparency, efficiency and quality in hospitals? BMJ 2013;346:f3197.

3 Department of Health. Delivering the NHS plan: next steps on investment, next steps on reform. United Kingdom: DH; 2002.

4 O'Dowd A. Coding errors in NHS cause up to $£ 1$ bn worth of inaccurate payments. BMJ 2010;341:c4734

5 Walker S. Capturing health information - a perspectives paper. HIM J 2006;35:13-22.

6 Shahid M, Tindall A. Payment by results: are we missing something? Int J Health Care Qual Assur 2013;26:387-91.

7 Nouraei SAR, Virk JS, Hudovsky A et al. Accuracy of clinician-clinical coder information handover following acute medical admissions: implication for using administrative datasets in clinical outcomes management. J Public Health (Oxf) 2016;38:352-62.

8 Heywood NA, Gill MD, Charlwood N et al. Improving accuracy of clinical coding in surgery: collaboration is key. J Surg Res 2016;204:490-5.

9 Tucker DM, Parry MC, Packham I. Financial ramifications of inaccurate clinical coding in two common orthopaedic procedures. The Bulletin of the Royal College of Surgeons of England 2016;98:347.

10 Dalal S, Roy B. Reliability of clinical coding of hip fracture surgery: implications for payment by results? Injury 2009;40:738-41.
11 Nouraei SA, O'Hanlon S, Butler CR et al. A multidisciplinary audit of clinical coding accuracy in otolaryngology: financial, managerial and clinical governance considerations under payment-by-results. Clin Otolaryngol 2009;34:43-51.

12 Beckley IC, Nouraei R, Carter SS. Payment by results: financial implications of clinical coding errors in urology. BJU Int 2009;104:1043-6.

13 Mitra I, Malik T, Homer J], Loughran S. Audit of clinical coding of major head and neck operations. Ann R Coll Surg Engl 2009;91:245-8.

14 Audit Commission. Introducing payment by results: getting the balance right for the NHS and taxpayers. London; 2005. Available at http://webarchive.nationalarchives.gov.uk/20150423154441/ http://archive.audit-commission.gov.uk/auditcommission/aboutus/ publications/pages/national-reports-and-studies-archive.aspx.html

15 Milburn JA, Driver CP, Youngson GG et al. The accuracy of clinical data: a comparison between central and local data collection. Surgeon 2007:5:275-8.

16 Colville RJ, Laing JH, Murison MS. Coding plastic surgery operations: an audit of performance using OPCS-4. Br J Plast Surg 2000;53:420-2.

17 Reddy-Kolanu GR, Hogg RP. Accuracy of clinical coding in ENT day surgery. Clin Otolaryngol 2009;34:405-7.

18 Cadieux DC, Goldszmidt M. It's not just what you know: junior trainees' approach to follow-up and documentation. Med Educ 2017;51:812-25.

19 Carr ER, Pillai A. Re: A multidisciplinary audit of clinical coding accuracy in otolaryngology: financial, managerial and clinical governance considerations under payment-by-results. Clin Otolaryngol 2009;34:259-60; author reply 260-1.

20 Haliasos N, Rezajooi K, O'Neill KS et al. Financial and clinical governance implications of clinical coding accuracy in neurosurgery: a multidisciplinary audit. Br J Neurosurg 2010;24:191-5.

21 Barker J. Interactive complexity and comorbidity splits in Health Resource Group 4+. J Healthc Manag 2015:21:433-9.

22 Charlesworth A, Hawkins L, Marshall L. NHS payment reforms: lessons from the past and directions for the future. London: Nuffield Trust, 2014.

23 2017/8 and 2018/9 National Tariff Payment System: a consultation notice. NHS England and NHS improvement: 2016.

24 Long W. Extracting Diagnoses from Discharge Summaries. AMIA Annu Symp Proc 2005;2005:470-4.

Address for correspondence: Dr Krsna Mahbubani, Clinical coding, Salton House, St Mary's Hospital, Praed Street, London W21NY, UK, Email: krsna.mahbubani@nhs.net 